Conclusions Very high levels $(42.1 \%)$ of $\mathrm{VH}$ persist in a predominantly Black SLE population. Despite lower vaccine uptake, 66.1\% with COVID-19 $\mathrm{VH}$ had a recent flu vaccine, indicating potential vaccine receptivity. With less trust in the government, news, doctors, and lupus groups, community leaders and peers should lead outreach. Focus should include those who are younger, Black, and from lower socioeconomic groups, particularly with depression.

\section{COVID-19 VACCINE BELIEFS AMONGST INDIVIDUALS LIVING WITH LUPUS}

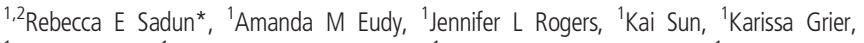
${ }^{1}$ Edna Scarlett, ${ }^{1}$ Mithu Maheswaranathan, 'Lisa G Criscione-Schreiber, 'Jayanth Doss, ${ }^{1}$ Megan EB Clowse. ${ }^{1}$ Duke University, Department of Medicine, Division of Rheumatology; ${ }^{2}$ Duke University, Department of Pediatrics, Division of Rheumatology

10.1136/lupus-2021-lupus21century.72

Background Patients with systemic lupus erythematosus (SLE) are known to be at higher risk for severe COVID-19. However, some individuals with SLE are hesitant to receive the COVID-19 vaccine. This study assessed the basis for COVID19 vaccine hesitancy in individuals with SLE.

Methods A survey was distributed electronically, through the Lupus Foundation of America (LFA) e-newsletters and social media channels, March through April of 2021. The survey was based on an adaptation of the Vaccine Hesitancy Scale (VHS), with additional questions specific to COVID-19 vaccines and detailed demographics.

Results A total of 415 people responded to the survey, of whom 91\% indicated that SLE was their primary rheumatologic diagnosis. Mean respondent age was 48, and $27.5 \%$ had less than a college education, whereas $31.3 \%$ had a graduate degree. When asked about race and ethnicity, $68.7 \%$ identified as non-Hispanic White, $15.2 \%$ as Black, 9.6\% as Latinx, $4.6 \%$ as Asian, and $1.2 \%$ as Native American. At the time of the survey, 59.6\% of respondents had received at least one COVID-19 vaccine dose; of these, $44.5 \%$ had spent less than 1 hour scheduling the vaccine, with $30.7 \%$ having spent $1-4$ hours and $24.8 \%$ having spent over 4 hours. Another 20.2\% of respondents wanted the COVID-19 vaccine but had not yet received it, and 20.2\% did not want the vaccine. The prior year, $76 \%$ of respondents received the flu shot; $16.1 \%$ of respondents indicated they don't trust the industry that creates vaccines. When asked about their beliefs regarding the COVID-19 vaccines, $66.6 \%$ were concerned the vaccine could flare their SLE, $27.1 \%$ were worried the vaccine could interact with their medications, $23.5 \%$ were concerned due to a history of allergies to foods and/or medications, and $12.2 \%$ were afraid they could get COVID-19 from the vaccine. Hesitant respondents were asked what would make them more likely to get the vaccine, and they cited evidence of safety in lupus as most important factor, with recommendations from the American College of Rheumatology (ACR), the LFA, or 'a trusted doctor' as more influential than vaccine mandates (figure 1).

Conclusions These results indicate an opportunity for healthcare professionals and patient advocacy organizations to assuage unfounded patient concerns, such as vaccine interactions with medications or food allergies being a contraindication to COVID-19 vaccination, while reassuring patients that vaccine-triggered flares are rare.

Acknowledgement We would like to thank the Lupus Foundation of American for their partnership in this research.

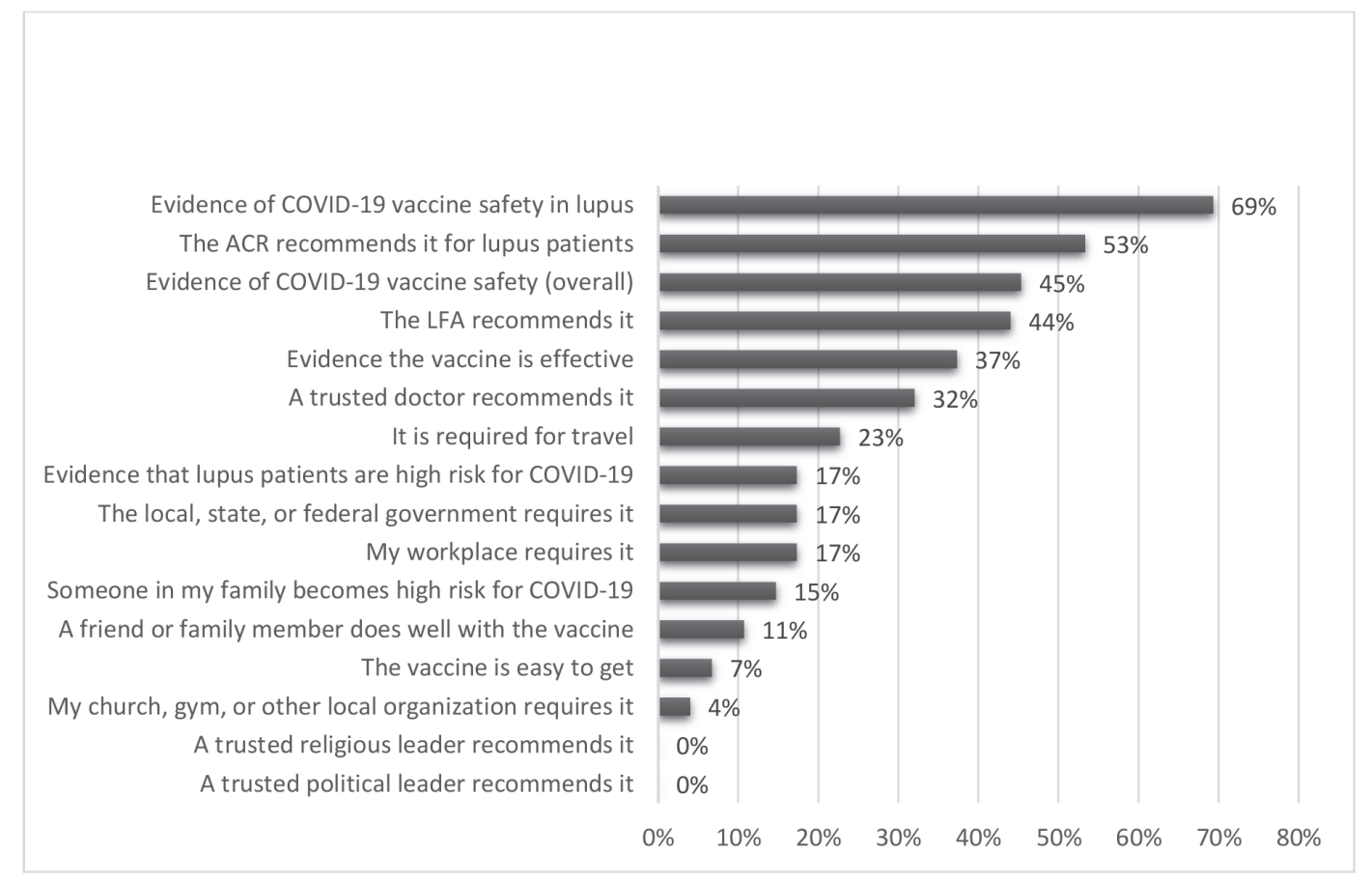

Abstract 1205 Figure 1 Respondents indicated which factors would have a positive influence on their likelihood of getting vaccinated against COVID-19; more than two-thirds of respondents indicated they would be more likely to pursue vaccination if they were presented with evidence of COVID-19 vaccine safety in lupus. 